Psychother Psychosom 2019;88:249-251

DOI: 10.1159/000500594

\section{Increasing Restlessness Signals Impending Increase in Depressive Symptoms More than 2 Months before It Happens in Individual Patients}

\author{
Arnout C. Smit Evelien Snippe Marieke Wichers
}

ICPE, Department of Psychiatry, University Medical Center Groningen, University of Groningen, Groningen, The Netherlands

Dear Editor,

Many patients are treated for their depressive symptoms with antidepressants. After discontinuation of all classes of antidepressants, the risk of depressive symptoms is higher than in patients who continue treatment [1-3]. However, tapering of antidepressants remains common in clinical practice because (a) many patients experience unwanted side effects; (b) prolonged use of antidepressants may worsen the long-term course of the depression [4, 5]; and (c) a substantial proportion of patients is able to taper without an increase in depressive symptoms [1-3]. Real-time monitoring of the risk of depressive symptoms during the tapering of medication may be useful. If the risk is increasing, tapering could be put on hold until the risk decreases, or stopped if the risk remains elevated.

A symptom that may be valuable for monitoring the risk of depressive symptoms is restlessness. The participant of the first study demonstrating early signs of an upcoming increase in depressive symptoms [6] (hereafter Patient 0 ) spontaneously mentioned that he noticed an increase in restlessness starting before the onset of core depressive symptoms (personal communication). Restlessness is also a defining symptom in generalized anxiety, the most frequently described symptom in prodromal phases of depression [7]. The aim of the current paper is to assess if a rise in ecological momentary assessments of restlessness during or after tapering of antidepressants can be found before an increase in depressive symptoms, but not in patients who tapered without such an increase.

The participants were 6 individuals with a history of depression who made a shared decision with their medical doctor to taper their antidepressants. These patients (1-6) filled in a maximum of 3 daily questionnaires using a 5.5-h fixed interval experience sampling protocol for 95-183 days during and after open-label tapering of their antidepressant (for the type of medication and tapering schedule, see Fig. 1). Patient 0 , whose data were already collected, was added to this sample. Patient 0 collected up to 10 daily questionnaires before, during, and after gradual tapering (for details, see [6]). Patients 1-6 answered the item "I feel restless" on a visual analogue scale (range: "not at all" to "very"); Patient 0 answered the same item on a 7-point scale.

Five participants tapered their medication without an increase in depressive symptoms. Patients 0 and 1 reported an increase in depressive symptoms. A nonparametric change point analysis (ecp $\mathrm{R}$ package; [8]) of the weekly assessed depression subscale of the Symptom Checklist-90 showed a significant $(p<0.005)$ increase in depressive symptoms, which coincided with the timing of the selfreported increase. It is unlikely that this reflects withdrawal effects, since (a) Patients 0 and 1 did not report physical withdrawal effects that impaired functioning in any way, and (b) depressive symptoms did not significantly increase until weeks after tapering was completed, which would be an unlikely timing for withdrawal symptoms [9]. Late-onset withdrawal disorders cannot be ruled out.

We used exponentially weighted moving average (EWMA) charts to detect structural changes in restlessness prospectively [10]. After each measurement of restlessness $\left(\mathrm{Y}_{\mathrm{t}}\right)$, a running estimate of the mean level of restlessness was updated using $\mathrm{EWMA}_{\mathrm{t}}=\lambda \times \mathrm{Y}_{\mathrm{t}}+(1-\lambda) \times \mathrm{EWMA}_{\mathrm{t}-1}$. The EWMA is a weighted average over available observations; the more recent an observation, the higher the associated weight. Parameter $\lambda$ controls the rate at which the weights decrease. To detect a potentially small increase in the mean, a low $\lambda$ was used $(\lambda=0.05)$. An even lower $\lambda=0.01$ was used for Patient 0 to smooth out some of the erratic behavior caused by the discrete answering scale. We controlled for autocorrelation if $A R(1)$ had a lower AICc than $A R(0)$ to prevent false alarms caused by high autocorrelation. To limit the influence of extreme values, data points $>3$ SDs from the mean of the first 100 observations were Winsorized. After each questionnaire, the updated EWMA was marked as potentially alarming if it exceeded a bandwidth calculated based on the first 100 observations $(L=3$; see [10] for technical details).

Results are presented in Figure 1. The EWMA charts demonstrate an alarming rise in restlessness more than 2 months before the increase in depressive symptoms of Patients 0 and 1 . No false alarms were found in the other patients.

Post hoc analyses showed that total negative affect and positive affect scores did not have the same capacity to signal future increase in depressive symptoms. EWMA charts showed (a) decreasing negative affect and increasing positive affect scores in Patient 0 before the increase in depressive symptoms, and (b) false alarms in 3 out of the 5 patients who did not show any increase in depressive symptoms (full results available upon request).

This study indicates that monitoring restlessness could be a valuable method in clinical practice for the early detection of impending increase in depressive symptoms because (a) it can provide risk assessment for individual patients rather than at a group level; (b) it can continuously update the risk assessment in real time while data is collected; and (c) the statistical implementation and

\section{KARGER}

E-Mail karger@karger.com www.karger.com/pps
(C) 2019 The Author(s)

Published by S. Karger AG, Basel

Karger

0 pen access

This article is licensed under the Creative Commons AttributionNonCommercial-NoDerivatives 4.0 International License (CC BYNC-ND) (http://www.karger.com/Services/OpenAccessLicense) Usage and distribution for commercial purposes as well as any distribution of modified material requires written permission.
Arnout C. Smit

ICPE, Department of Psychiatry

University Medical Center Groningen, University of Groningen

Hanzeplein 1, NL-9713 GZ Groningen (The Netherlands)

E-Mail a.c.smit@umcg.nl 


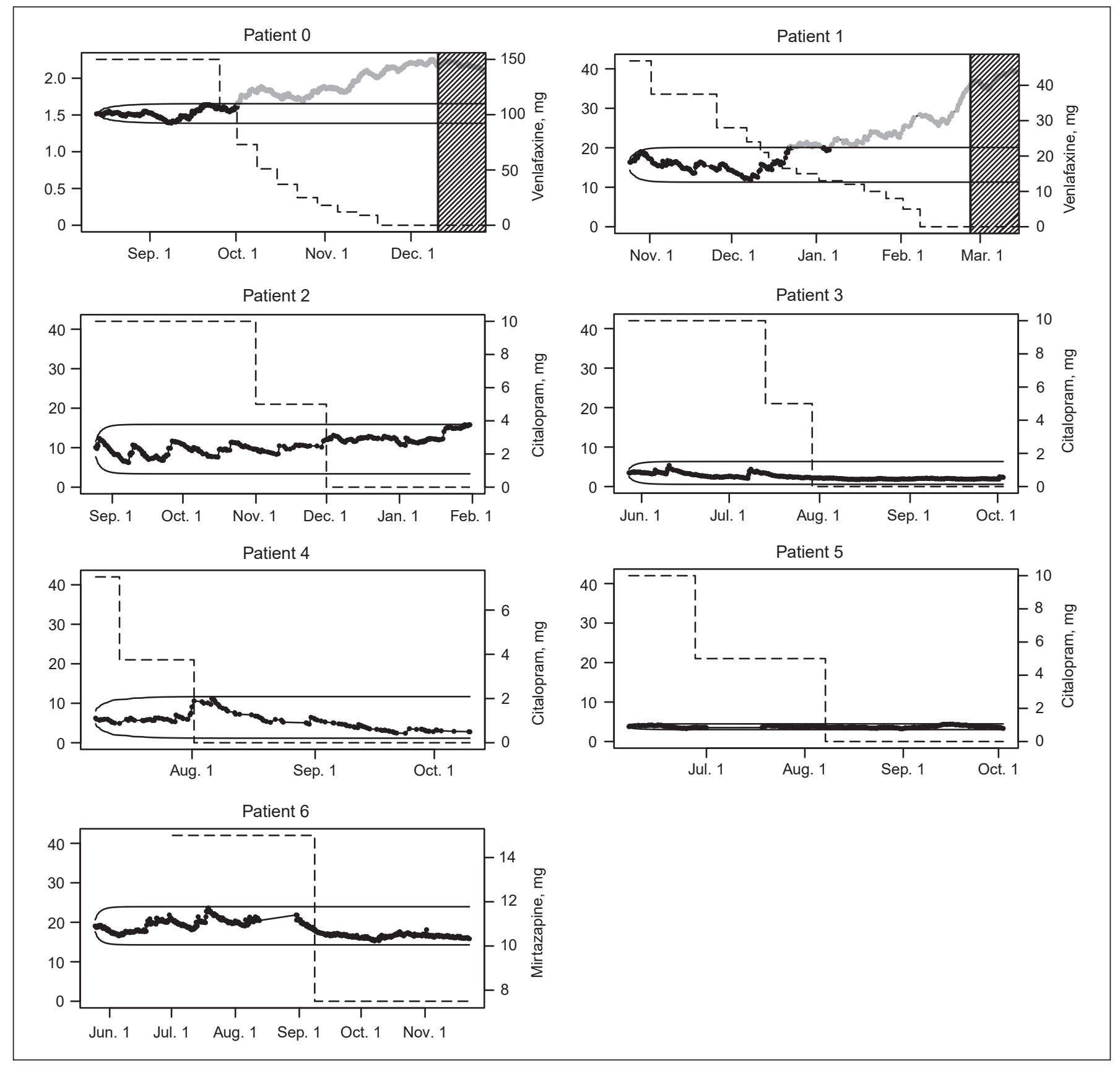

Fig. 1. EWMA charts of self-reported restlessness over time in patients tapering their antidepressants. Black dots represent the EWMA of restlessness scores (scales are indicated on the left vertical axes). Dots are colored grey if the EWMA was higher than would have been expected given the process has not changed com-

pared to the first 100 observations. The dashed line represents the dosage of the tapered antidepressant when known (scales and antidepressant names are indicated on the right vertical axes). Charts of Patients 3 and 6 were controlled for autocorrelation; for the other patients, the regular EWMA charts are reported.

interpretation are relatively straightforward. This allows clinicians and patients to reevaluate the tapering schedule every time a new questionnaire is filled in.

Considering the consistent results, it seems unlikely that the findings are caused by chance alone, despite the small sample size.

However, replication is warranted, and future studies with a larger sample are needed to assess to whom these results generalize. Specifically, it should be studied if restlessness also signals an upcoming increase in depressive symptoms in individuals who tapered other types of medication than venlafaxine. 
Current data provide no insight in why restlessness increased in Patients 0 and 1. As hypothesized, restlessness may have increased as prodrome of depression. An alternative explanation is that the increase in restlessness was a withdrawal effect of the medication, suggesting that Patients 0 and 1 may have been more vulnerable to experience an increase in depressive symptoms because of withdrawal effects. Though more research in this area is needed, the ability to forecast a future increase in depressive symptoms is promising for clinical applications regardless of the mechanism.

\section{Acknowledgement}

The authors thank the participants for their time and effort, the clinicians at the Center for Integrative Psychiatry (Lentis), and F.M. Bos for their assistance in the recruitment of participants, and P.C. Groot for his input on the study design and for data collection.

\section{Statement of Ethics}

The authors assert that all procedures contributing to this work comply with the ethical standards of the relevant national and institutional committees on human experimentation and with the Helsinki Declaration of 1975, as revised in 2008. All participants provided written informed consent.

\section{Disclosure Statement}

The authors have no conflicts of interest to declare.

\section{Funding Sources}

This project has received funding from the European Research Council (ERC) under the European Union's Horizon 2020 research and innovative programme (ERC-CoG-2015; No. 681466 to M.W.).

\section{Author Contributions}

A.C.S. formulated research questions, designed and performed the data analysis, interpreted the results, and wrote the manuscript.
E.S. designed the data collection method, was responsible for data collection, formulated research questions, interpreted the results, and revised the manuscript. M.W. designed the data collection method, formulated research questions, interpreted the results, and revised the manuscript.

\section{References}

1 Geddes JR, Carney SM, Davies C, Furukawa TA, Kupfer DJ, Frank E, et al. Relapse prevention with antidepressant drug treatment in depressive disorders: a systematic review. Lancet. 2003 Feb;361(9358):653-61.

2 Glue P, Donovan MR, Kolluri S, Emir B. Meta-analysis of relapse prevention antidepressant trials in depressive disorders. Aust N Z J Psychiatry. 2010 Aug;44(8):697-705.

3 Sim K, Lau WK, Sim J, Sum MY, Baldessarini RJ. Prevention of relapse and recurrence in adults with major depressive disorder: systematic review and meta-analyses of controlled trials. Int J Neuropsychopharmacol. 2015 Jul;19(2):pyv076.

4 El-Mallakh RS, Gao Y, Jeannie Roberts R. Tardive dysphoria: the role of long term antidepressant use in-inducing chronic depression. Med Hypotheses. 2011 Jun;76(6):769-73.

5 Fava GA, Offidani E. The mechanisms of tolerance in antidepressant action. Prog Neuropsychopharmacol Biol Psychiatry. 2011 Aug;35(7): 1593-602.

6 Wichers M, Groot PC; Psychosystems, ESM Group, EWS Group. Critical slowing down as a personalized early warning signal for depression. Psychother Psychosom. 2016;85(2):114-6.

7 Fava GA, Tossani E. Prodromal stage of major depression. Early Interv Psychiatry. 2007 Feb;1(1):9-18.

8 James NA, Matteson DS. ecp: an R package for nonparametric multiple change point analysis of multivariate data. J Stat Softw. 2014 Jan;62(7): $1-25$.

9 Chouinard G, Chouinard VA. New classification of selective serotonin reuptake inhibitor withdrawal. Psychother Psychosom 2015 Mar;84(2): 63-71.10 Montgomery DC: Introduction to Statistical Quality Control. 6th ed. New York: John Wiley \& Sons; 2009.

10 Montgomery DC. Introduction to Statistical Quality Control. 6th ed. New York: John Wiley \& Sons; 2009. 